\title{
DIRECT NANOSCALE MAPPING OF OPEN CIRCUIT VOLTAGES AT LOCAL BACK SURFACE FIELDS FOR PERC SOLAR CELLS
}

\author{
Alexandra Longacre ${ }^{1}$, Michael Martin ${ }^{1}$, Thomas Moran ${ }^{1}$, Oleg V. Kolosov ${ }^{2}$, Eric Schneller ${ }^{3}$, \\ Alan J. Curran ${ }^{4}$, Menghong Wang ${ }^{4}$, Jianfang Dai ${ }^{5}$, Laura S. Bruckman ${ }^{4}$, Jean-Nicolas Jaubert ${ }^{6}$, \\ Kristopher O. Davis ${ }^{3}$, Jennifer L. Braid ${ }^{4}$, Roger H. French ${ }^{4}$, Bryan D. Huey, ${ }^{1, *}$ \\ 1) University of Connecticut, Storrs, CT, USA. \\ 2) Lancaster University, Lancaster, UK. \\ 3) University of Central Florida, Orlando, FL, USA. \\ 4) Case Western Reserve University, Cleveland, Ohio, USA. \\ 5) Cybrid Technologies, Suzhou City, Jiangsu, China. \\ 6) Canadian Solar Inc., Suzhou, Jiangsu, China. \\ *Corresponding Author: bryan.huey@uconn.edu
}

\begin{abstract}
The open circuit voltage $\left(V_{O C}\right)$ is a critical and common indicator of solar cell performance as well as degradation, for panel down to lab-scale photovoltaics. Detecting $V_{O C}$ at the nanoscale is much more challenging, however, due to experimental limitations on spatial resolution, voltage resolution, and/or measurement times. Accordingly, an approach based on Conductive Atomic Force Microscopy is implemented to directly detect the local $V_{o c}$, notably for monocrystalline Passivated Emitter Rear Contact (PERC) cells which are the most common industrial-scale solar panel technology in production worldwide. This is demonstrated with cross-sectioned monocrystalline PERC cells around the entire circumference of a poly-Aluminum-Silicide via through the rear emitter. The Voc maps reveal a local Back Surface Field extending $2 \mu \mathrm{m}$ into the underlying p-type Si absorber due to Al in-diffusion as designed. Such high spatial resolution methods for photovoltaic performance mapping are especially promising for directly visualizing the effects of processing parameters, as well as identifying signatures of degradation for silicon and other solar cell technologies.
\end{abstract}

Keywords-Conductive Atomic Force Microscopy, solar cells, photovoltaics, PERC, Back Surface Field. 


\section{INTRODUCTION}

With ever-expanding global power consumption, and practical and societal imperatives to increase the fraction of renewable energy systems such as solar, there is a clear necessity for optimizing the efficiency, lifetime, and reliability of photovoltaic (PV) power generation systems across the commercial and consumer sectors. Furthermore, economic pressures continually demand improved performance and diminished manufacturing costs. These expectations require assessment at the system, panel, module, and even finer length scales. For solar power stations, drone surveys are becoming increasingly practical [1]. At the panel and module level, macroscopic current voltage measurements through the fully assembled electrodes yield measures of the short circuit current $\left(\mathrm{I}_{\mathrm{sc}}\right)$, open circuit voltage $\left(\mathrm{V}_{\mathrm{OC}}\right)$, maximum power $\left(\mathrm{P}_{\mathrm{MP}}\right)$ points such as the current and voltage at max power (IMP and $V_{M P}$, respectively), intensity sensitivity such as 'Suns- $V_{o c}$ ' measurements [2], etc. With spatial resolution down to the micrometer-scale, optical measurements have also become ubiquitous, often as a function of wavelength, including Suns-Photoluminescence [3]. Commonplace approaches include detecting or mapping absorption and reflection, electroluminescence, or photoluminescence, providing crucial PV performance indicators to assess the quantum efficiency, recombination rates, and photocarrier lifetimes.

Nanometer-scale investigations, however, can still be challenging due to limitations in resolution, measurement speed, and in-situ compatibility. Promising nanoscale approaches for the most common markers of solar cell performance are primarily based on Atomic Force Microscopy [4]. For instance, Photo-Conducting AFM (pcAFM) records pA to nA scale currents between a solar cell and a conducting AFM tip which serves as a nanometer-scale constantlypositionable local electrode. This enables local measurements and direct mapping via tip scanning of $I_{s c}$ as well as intensity or wavelength sensitivities, all as a function of local microstructure, concentration gradients, or grain boundaries and other interfaces. Experimental approaches for $V_{o c}$, on the other hand, most commonly Scanning Surface Potential Microscopy $[5,6]$ as well as pcAFM-based I/V spectroscopy [7], provide valuable insight but often necessitate non-contact detection or multi-pass imaging, respectively with concomitant limitations such as diminished voltage resolution and usually relatively slow measurement acquisition. Accordingly, a method for direct local $V_{o c}$ mapping is implemented based on continuously updating the bias necessary to achieve null photocurrents, notably identical to the actual measurement conditions for up to grid-scale $V_{0 c}$ assessments.

While essentially every solar-cell technology can benefit from such high-resolution local investigations of $\mathrm{V}_{\mathrm{OC}}$ and other PV performance markers, this work focuses on Passivated Emitter Rear Contact cells due to their dramatic recent market penetration. PERC has seen 
widespread adoption by manufacturers largely due to its relatively straightforward implementation with increased processing steps, a negligible increase in necessary infrastructure, but a marked bump in efficiency [8]. The concept is based on a conventional Al doped Back-Surface-Field p-type solar cell, except instead of a continuous back electrode, the rear contact is largely passivated in order to slightly but non-negligibly diminish carrier recombination. Technically, before a conventional rear contact metallic paste is fired into place, a continuous passivating layer is first grown (typically oxides and/or nitrides). Pulsed laser processing is then patterned and/or rastered to locally melt and recrystallize distinct conducting vias that punch through the passivation layer and furthermore diffuse Al into the underlying p-type Si to generate local instead of continuous back surface fields [9].

While PERC is effective for increasing cell efficiency by a market-worthy $~ 0.1 \%$, the vias in the rear contact are hypothesized to be likely regions for the earliest stages of $P V$ degradation. This may be due to the different processing steps, different local operating conditions, and/or higher current densities in these localized back surface fields with a corresponding increase in local temperature [10]. This may manifest as differences in initial [11] or long term stability, and could be compounded by distinct degradation profiles for multi-crystalline compared to single crystal silicon cells [12]. Moreover, the local BSF's become possible sites for preferential ingress of damaging chemical species. This may result from any intensity- or temperature- induced long-term breakdown of ethylene-vinyl-acetate or related packaging components [13-16]. It is certainly accelerated by exposure to water, humidity, and salt exposure $[17,18]$. In order to engineer optimal devices, it is therefore imperative to investigate local degradation effects [19].

\section{EXPERIMENTAL APPROACH}

\section{A. Materials and Equipment}

The monocrystalline PERC cells investigated were prepared by the DuPont Silicon Valley Technology Center using conventional methods and implementing their Solamet metallization technology [20]. Specifically the cells are based on M2 sized Longi p-type silicon wafers, with a thickness of $170 \mu \mathrm{m}$ and base resistivity of $2.25 \Omega * \mathrm{~cm}$. The front side features lightly doped emitter LDE style $n$-type diffusions ( $75 \Omega / \mathrm{sq}$ ) to optimize $\mathrm{V}_{\text {oc }}$ by limiting recombination [21]. The back side, investigated in this work, features polycrystalline aluminum silicide PERC vias (80 $\Omega / s q)$.

These specimens are designed with a pattern of linear local BSF's on a $1 \mathrm{~mm}$ period, instead of a 2-dimensional array of point contacts. The rear surface has a continual overlying metal paste, as this is a monofacial instead of a bifacial design where patterend rear electrode lines would be present as they are on the front surface. This specimen configuration is particularly 
amenable to cross sectional studies, as multiple local BSF's can be revealed in a single sample preparation setp and investigated locally through single electrode contacts. We employed shallow angle cross sectioning by conventional multi-beam argon ion polishing (Leica EM TIC020 triple ion-beam cutter), minimizing ion damage through tungsten shielding, in order to expose 5-8 linear local BSF regions during each ion-polishing attempt. SEM and EDS to investigate the composition for a fracture-cross-sectioned PERC from an identical specimen via are acquired with a Thermo Fisher Teneo LoVac SEM equipped with an EDAX EDXS system.

Nanoscale measurements are performed using a customized AFM (Asylum Research mfp-3D, with ORCA integrated picoammeter) mounted on an inverted optical microscope platform (Nikon TE-2000). This enables in-situ front-surface light exposure, in this case using a CREE high performance broadband LED, but which can practically couple with any solar simulator, bandfiltered wavelengths, laser line, etc. The pcAFM probe serves as a local and scannable rear contact, while also maintaining force feedback and thereby mapping the local surface topography with nanoscale resolution in all dimensions. Typical stable imaging sizes are only $\sim 5$ $\mu \mathrm{m}$, however, due to the specimen tilt and limitations set by the maximum out-of plane (z) scan range. In order to visualize substantially larger areas, montages of multiple scans purposefully acquired with moderate overlaps ( $10 \%)$ are assembled in post-processing based on standard feature recognition and image alignment routines (ImageJ). Specifically, a rigid transformation and/or rotation (uniform $x, y$ shift or image reorientation) is employed based purely on the topography data, and then these image transformations are subsequently applied to the direct Voc data as well.

\section{B. Direct Voc mapping}

Using a conducting AFM probe and fine current detection (Fig. $1 \mathrm{~A}$ ), it is already possible to locally investigate photoconduction at any location of interest-for instance at the local BSF for a shallow angle polished PERC cell (B). Individual current/Voltage (I/V) curves, idealized in (C), or often grids of I/V spectra, can then be acquired and later analyzed for various performance parameters or statistics. Notably, the cell is mounted 'upside down' in the inverted optical microscope, and also depicted this way in the schematic as well as all results to follow (i.e. the 'back electrode' is at the top of the field of view). The front face is therefore illuminated as usual, while the AFM probe interrogates the local photoresponse at the rear electrode and especially for exposed regions beneath that were otherwise buried before cross-sectioning. In this manner, the photovoltaic performance can be investigated as a function of position, distance from a certain interface, microstructural phase, or other segmentable region. However, this all necessitates robust data acquisition that is spatially and temporally artifact 
free, a substantial challenge given the often long practical measurement times for high resolution PV performance mapping.

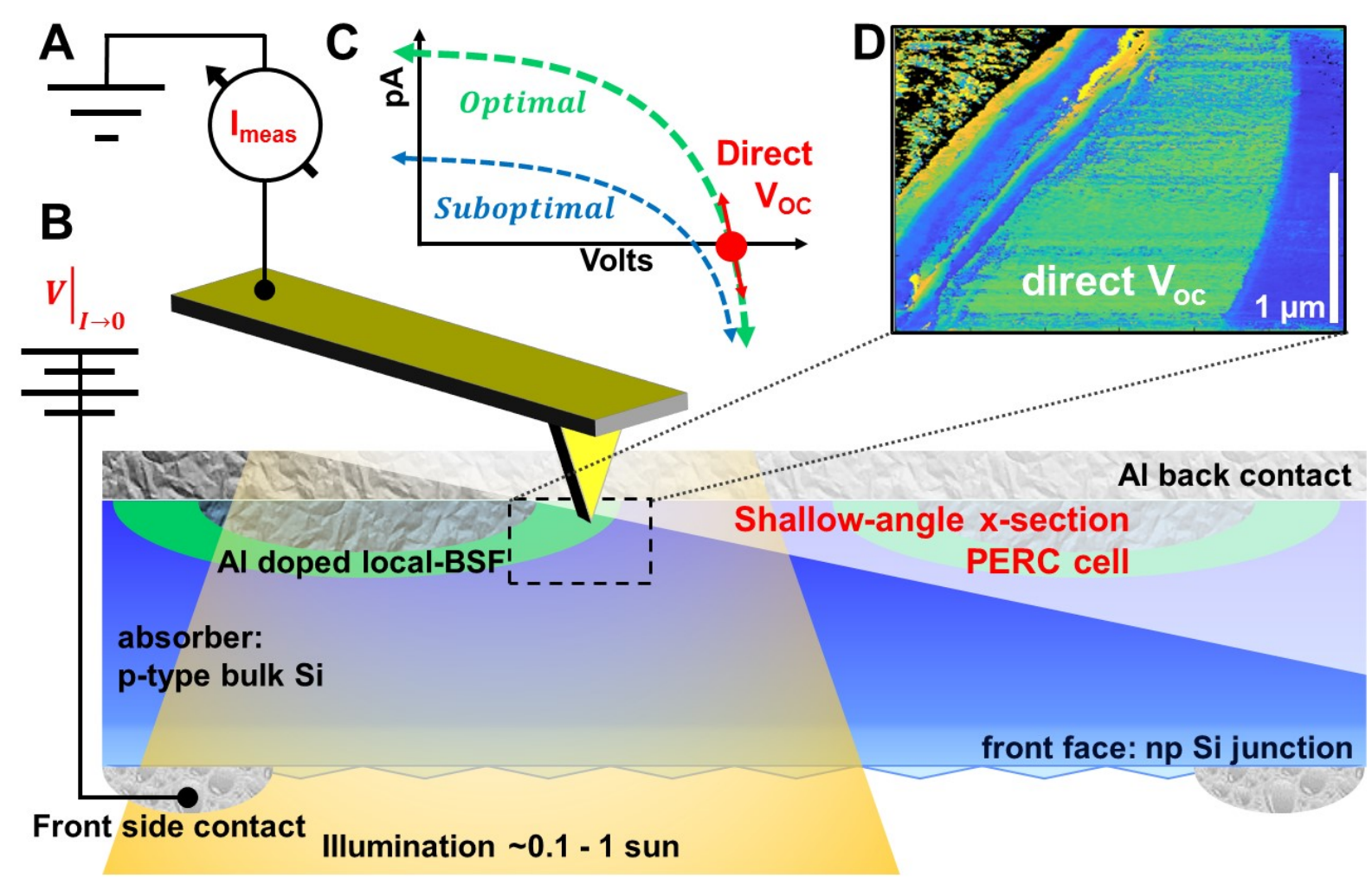

Fig. 1: Experimental schematic, implementing (A) photoconducting AFM of a (B) shallowangle cross-sectioned passive emitter rear contacts (PERC) cell. The bias between the patterned front side contact and the grounded conducting AFM probe is automatically and continually adjusted to (C) maintain a null photocurrent in order to directly measure the open circuit voltage (Voc). By scanning in the vicinity of a via through the rear passive emitter (D), the local back-surface field can be directly and efficiently mapped (D).

As a result, we developed a method to directly acquire maps of $V_{O C}$ by mimicking conventional open circuit potential mapping, except at typical AFM-probe dimensions. This is based on null currents (D), using an extra custom-configured proportional-integral-derivative feedback loop during otherwise normal contact-mode AFM operation. This additional feedback system constantly updates the bias applied to the front surface contacts, in order to null the photocurrent and thereby locally maintain (and record) open circuit conditions [22]. This is an important advance, as previous efforts to map parameters such as the open circuit voltage or maximum power points generally require necessitate slow and sometimes artifact-inducing 
procedures. These require the acquisition of either thousands of individual I/V spectra, or at least tens or hundreds of images each in a single location but using a range of biasing conditions [23].

\section{RESULTS AND ANALYSIS}

Fig. 2A presents optical microscopy of the rear electrode and shallow-angle-polished crosssection, which progressively exposes sub-surface components from top to bottom of the image. Each vertical dark band results from laser processing during manufacturing to generate the (in this case linear) PERC vias. These are especially apparent upon further optical magnification (Fig. 2B) as highly reflective pseudo-hemispherical features, which result from their polycrystalline and more metallic behavior following local recrystallization as aluminum silicide. While sometimes exhibiting pores [24], or developing voids once the often compressed via is exposed, vias far from such macroscale flaws are still relatively rough topographically even after shallow-angle ion polishing. This is because the various grain orientations preferentially polish at different rates due to anisotropic mechanical properties and/or channeling effects, thereby leaving an apparently faceted surface structure. However, at the interface between each via and the surrounding single crystal $\mathrm{Si}$, the surface is sufficiently smooth for relatively artifactfree AFM investigations such as photocurrent mapping. Fig. $2 \mathrm{C}$ displays Isc (simply the photocurrent upon illumination at 0 bias, with respect to zero photocurrent under dark conditions), while (D) depicts the directly detected $V_{O C}$ at this interface. Each reveals contrast, extending 1 to $2 \mu \mathrm{m}$ from the poly-Si via, which indicates an Al-doped $\mathrm{p}+$ region. This results from Al diffusion while the polycrystalline metallic via is forming, establishing the $p / p+$ localized BSF that is critical to the PERC technology since the remaining back electrode and monocrystalline silicon interface is in fact electrically disconnected by the nanoscale-thickness passivating barrier. 


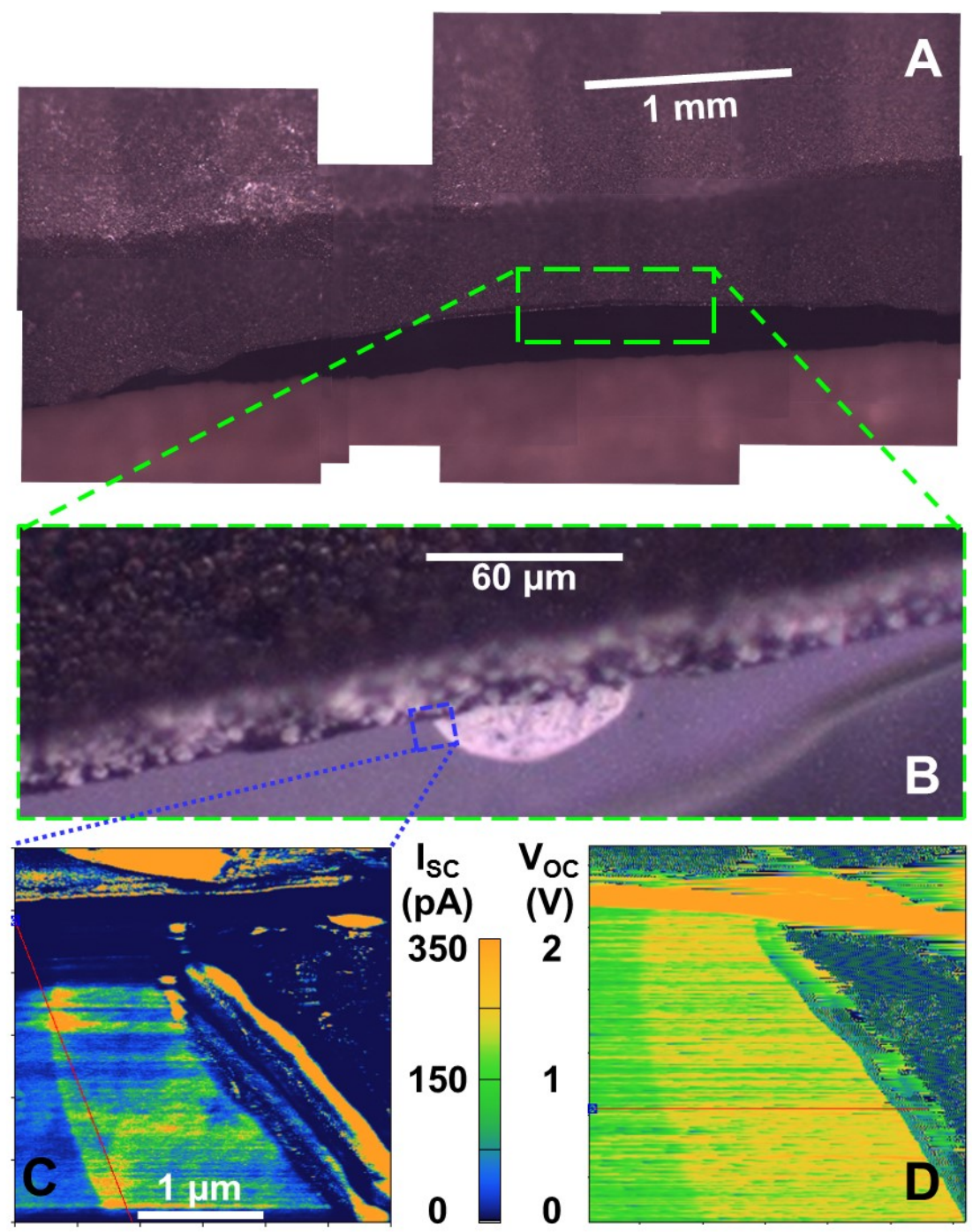

Fig. 2: Optical images of a shallow-angle-cross-sectioned PERC cell under progressive magnification as noted, (A) through the continuous back electrode to expose several rear contact lines (stripes), and (B) revealing one of several vias through the continuous passive emitter (B). At the corners of such poly-Si local electrode regions, the directly measured Isc (C, from AFM) and also $V_{O C}(D$, new AFM approach described herein) reveal contrast indicative of the local BSF formed by in-diffused Al extending 1-2 $\mu \mathrm{m}$ into the bulk Si.

In addition to the clear evidence of the local BSF regions (increased band at center), some regions of the images in Figs. 2C-D are marred by artifacts which merit further discussion. These principally result from significant topography in the field of view, as the sample is mounted flat for optimal absorption of the illumination source from below, but therefore the tip is in fact scanning down a relatively substantial slope even for such a shallow-angle section $\left(\sim 15^{\circ}\right)$. 
Furthermore, the initial roughness of the polycrystalline rear electrode paste (at the top of the field of view) is retained, leading to substantial artifacts near the junction with the silicon below. Current probed directly at this back electrode, or within the polycrystalline aluminum silicide (at right of each image) can additionally be impacted by geometric artifacts. As already mentioned, preferential ion polishing of certain grains in the silicide can lead to major topographic features and corresponding current artifacts. In fact, the broad z-range necessary to display the full height data is not instructive. Focusing on the deflection (scanning error) signal, however, reveals the complex surface topography present, as depicted in Fig. 3 as a montage of over 30 such adjacent deflection images. Many distinct facets (regions of uniform contrast) are apparent, suggesting distinct grain orientations, supporting the expected polycrystallinity for such laser-processed PERC vias [9].

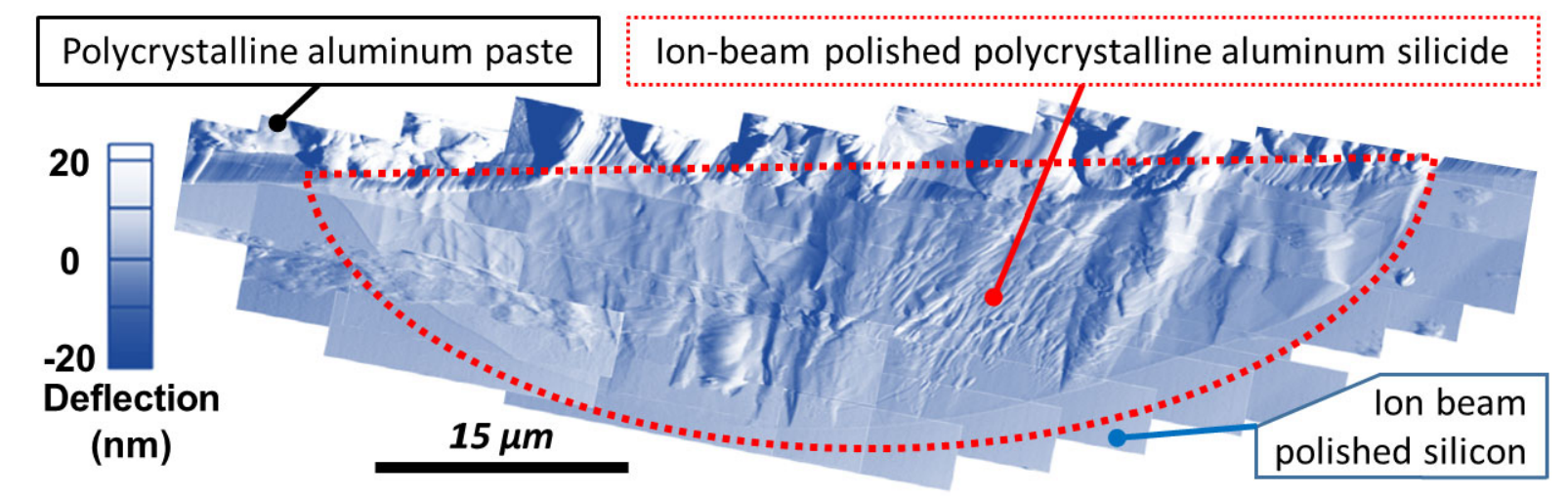

Fig. 3: AFM deflection images across an entire shallow-angle-cross-sectioned local BSF

Corresponding photocurrent images for this entire polycrystalline via (not shown for brevity) are inconclusive for 2 reasons. First, tip-sample artifacts can cause the measured current to drop to $0 \mathrm{pA}$, generally due to a local loss of tip-sample contact. This is usually caused by abrupt changes in sample slope, especially for major depressions in the surface resulting from pores in the particulate back electrode or highly preferentially ion-polished regions within the silicide. Second, method artifacts can occur when the surface is more smooth, but the tip is directly probing the electrically interconnected back-electrode and/or almost equally conducting polycrystalline silicide itself. In these cases, the measured currents frequently jump off-scale for the picoammeter built into the AFM tip holder (the results are clipped beyond 350 pA in Fig. 2, but practically currents as high as $20 \mathrm{nA}$ can be measured before current-limiting by built-in protective circuitry). In these instances, the actual photocurrents are almost certainly much higher. This is because photocarriers would in fact be collected or emitted along the entire back electrode, instead of just at any given nanoscale $(x, y)$ position contacted by the scanning AFM tip. The resulting poor data on conductors is an acknowledged limitation of our direct $V_{O C}$ 
mapping method, where the time constant for the current limiting circuitry is relatively slow compared to the null-current approach and hence the imaged $V_{O C}$ signal becomes pure noise or gets pegged at a null or extreme bias, as evident in the affected top and right sides of Fig. 2D.

Nevertheless, the concepts outlined in Figs. 1 and 2 are still valuable around and beneath the electrode and/or via, i.e. some of the regions of greatest concern for ultimate device performance and reliability. To achieve such direct, high resolution mapping of the magnitude and spatial extent of $\mathrm{V}_{\mathrm{OC}}$, for such regions of interest and/or illumination conditions (e.g. intensity and/or or wavelength), we remounted the specimen at a slight angle $\left(\sim 10^{\circ}\right)$ to physically avoid the via and back electrode as much as possible. This optimized the AFM-based imaging, but somewhat sacrifices absorption efficiency due to a slightly off-normal illumination angle. While this represents another acknowledged experimental limitation if true $V_{O C}$ measurements are required under standard illumination conditions and intensities, in practice the relative photocurrents are almost always far more relevant for nearly any AFM-based measurements [4].

The resulting Fig. 4A displays a montage of direct $V_{O C}$ images assembled from 21 individual scans, in order to demonstrate the method's stability and efficacy over large areas based on serial image/step/repeat processes followed by digital post-processing. This would be experimentally impractical if each of the incorporated individual maps themselves required numerous supporting images or thousands of I/V spectra. The data in Fig. 4A also confirms a possibly surprising detail apparent in Fig. 2D as well, that is the presence of a relatively uniform open circuit voltage within the local back surface field around every via. One might assume that a gradient should be present instead, except that $V_{O C}$ should be sensitive to the doping distribution and this is nearly constant across the Al-doped region surrounding each via. This results from the extremely rapid pulsed-laser melting and subsequent resolidification upon effectively quenching by the thermal mass of the surrounding silicon. 


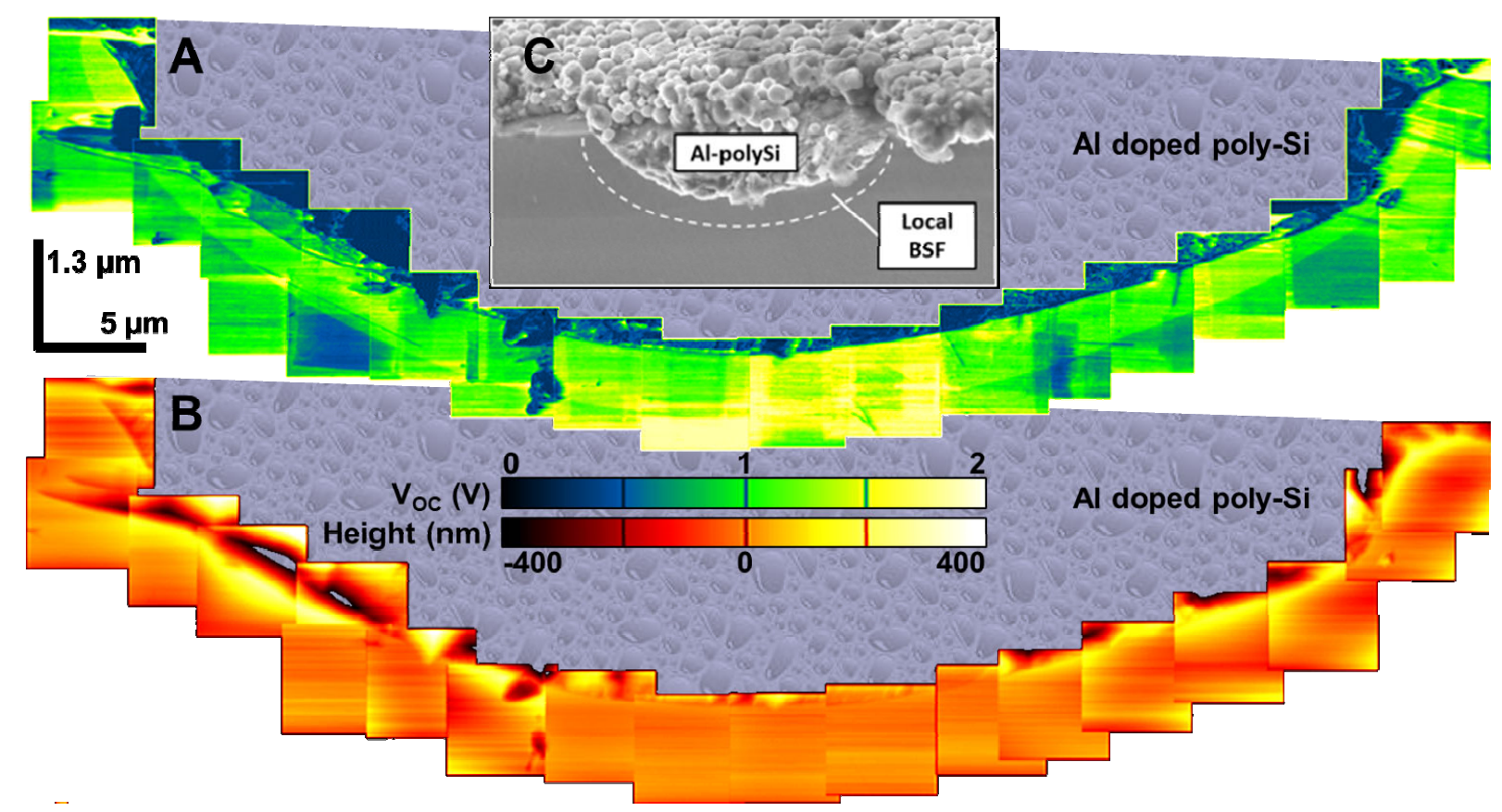

Fig. 4: Montage of 20 direct $V_{o c}$ maps around the circumference of an entire Al-doped poly-Si via through the rear surface passive emitter $(A)$. This reveals what appears to be $a \sim 2 x$ variation in the spatial extent of the local BSF, but after correcting for the shallow angle crosssection geometry is in fact a nearly uniform back surface field depth. The simultaneously acqurired topography images $(B)$ acquired simultaneously to $(A)$ reveal the edges of distinct grains of the polycrystalline aluminum silicide via, preferentially milled to various depths beneath the polished plane of the surrounding single-crystal silicon. A representative SEM fracture-cross-section (C) displays such a metal paste back electrode, poly-aluminum silicide via through the passive emitter, and an overlain schematic of the extent of the doped local BSF which is uyniquely apparent in (A).

Overall, these images map $V_{O C}$ around the entire circumference of a representative PERC via. The simultaneously acquired AFM topography (Fig. 4B) confirms again that the via comprises polycrystalline $\mathrm{Si}$ which is ion-polished at distinct rates depending on the locally recrystallized orientation, forming pits of distinct depths. This is generally confirmed by SEM images of fracture cross-sections from sister samples ( $C$, image courtesy Dupont). Within the bulk Si crosssection, which is orders of magnitude smoother than the via (where the rms roughness is $<5$ nanometers), the doped $V_{0 c}$ region appears to vary between $\sim 1-5 \mu \mathrm{m}$. It appears narrowest near the electrode, and extends furthest when deep beneath the original passivation layer/electrode interface. Once calibrating for the sample tilt due to shallow angle polishing, 
however, in fact the extent of this local back surface field is nearly equivalent around the whole via.

Fig. 5 displays scanning electron microscopy, and energy dispersive ( $x$-ray) spectroscopy and mapping results, for an identical via to those considered throughout this work. The composition map for a fracture cross-section (A) displays by contrast the presence of 4 chemical species ( $\mathrm{Si}$, $\mathrm{Al}, \mathrm{O}$, and $\mathrm{C}$ ), as noted in the legend (B). Identified by their $\mathrm{K}_{\alpha}$ peaks, a summary of their relative weight percentage across the field of view unsurprisingly reveals $\mathrm{Si}$ and $\mathrm{Al}$ are predominant. $\mathrm{A}$ corresponding secondary electron image (C), along with a schematic (D), displays the region considered. The composition map, along with the sum spectrum of emitted $x$-rays for the entire analyzed area (E) and especially the qualitatively similar spectrum (F) from the via alone (dotted region in $\mathrm{A}$ ), confirms a mixture of $\mathrm{Si}$ and $\mathrm{Al}$ in pockets with varying ratios within the via, indicative of recrystallization. The overlaying aluminum-paste reveals minor oxygen and carbon species, as well as some Si clusters, again often in pockets as expected based on the cell fabrication procedure.
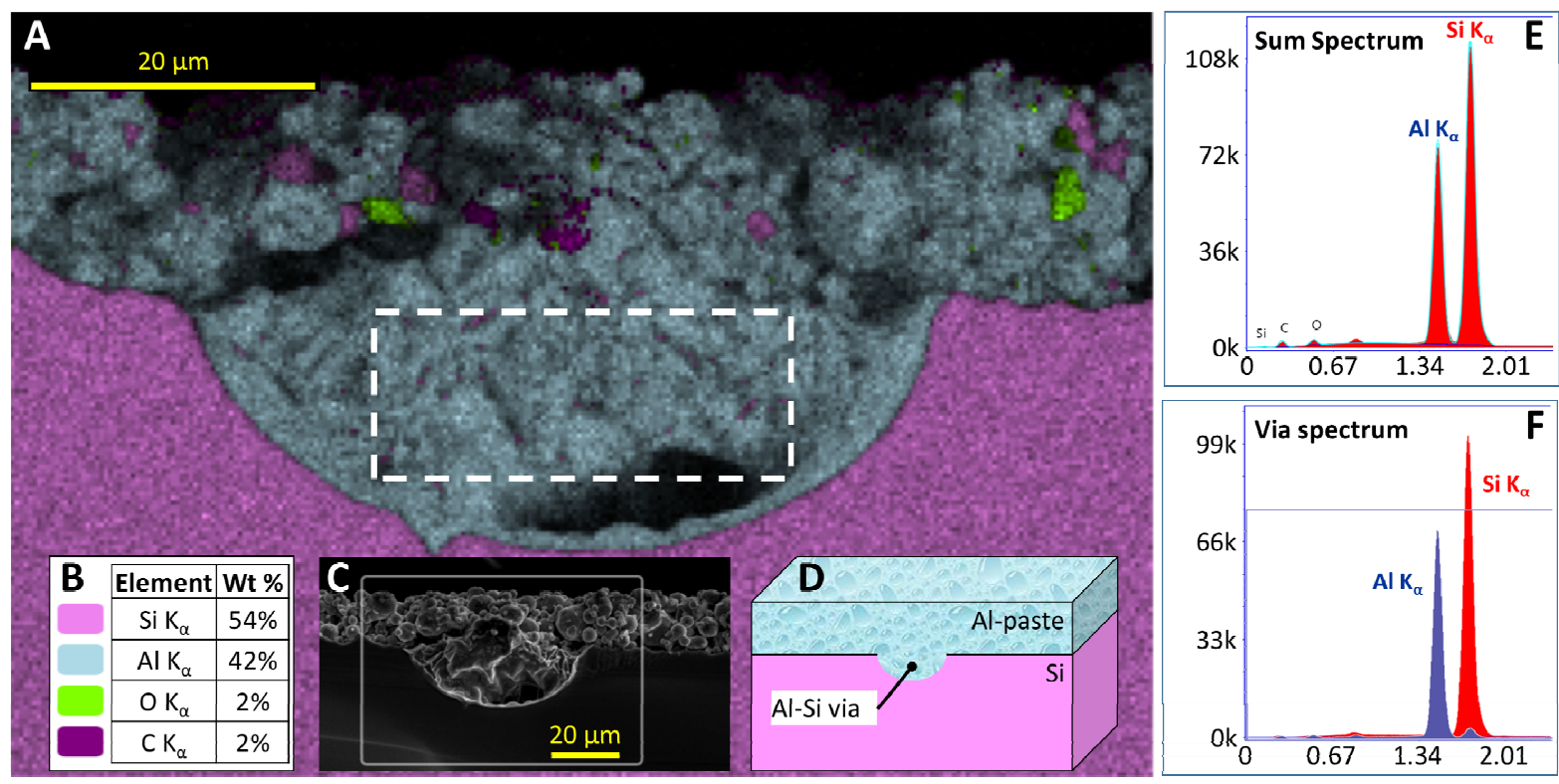

Fig. 5: Chemical analysis of via cross sesction: EDS composition map $(A)$, composition legend and calculated weight percentage for the overall field of view (B), secondary electron image of the analyzed fracture surface $(C)$, schematic $(D)$, sum x-ray spectrum for the mapped region $(E)$, and spectrum within the via alone (dashed region, $F$ ).

Finally, it is noteworthy that it is possible to reconfigure the measurement approach in Fig. 1 so that the extra feedback loop directly maps and analyzes variations in the maximum power point $\left(\mathrm{P}_{\mathrm{MP}}\right)$ instead of $\mathrm{V}_{\mathrm{OC}}$. This could reveal the corresponding local voltage and current under max power conditions as well ( $\mathrm{V}_{\mathrm{MP}}$, and $\mathrm{I}_{\mathrm{MP}}$, respectively). This is suggested by Fig. 6 , which is 
from simpler but not-spatially-resolved collections of power versus bias spectra. The overall collection of power vs. bias data (B) depicts the ensemble response at and around vias similar to those in Figs. 2-4. There are a few distinct peaks in power, at least for a small fraction of the overall collected spectra. Many others (zero contrast across the entire voltage range) were nulled out in post-processing because of poor tip-sample electrical contact, off-scale currents, etc., which without mapping cannot be further assessed. Any regions that under- or outperform their neighbors - such as those potentially signified by peaks in the various histograms of Figs. 6A, C, and D-could thus also be spatially identified instead of simply statistically analyzed.
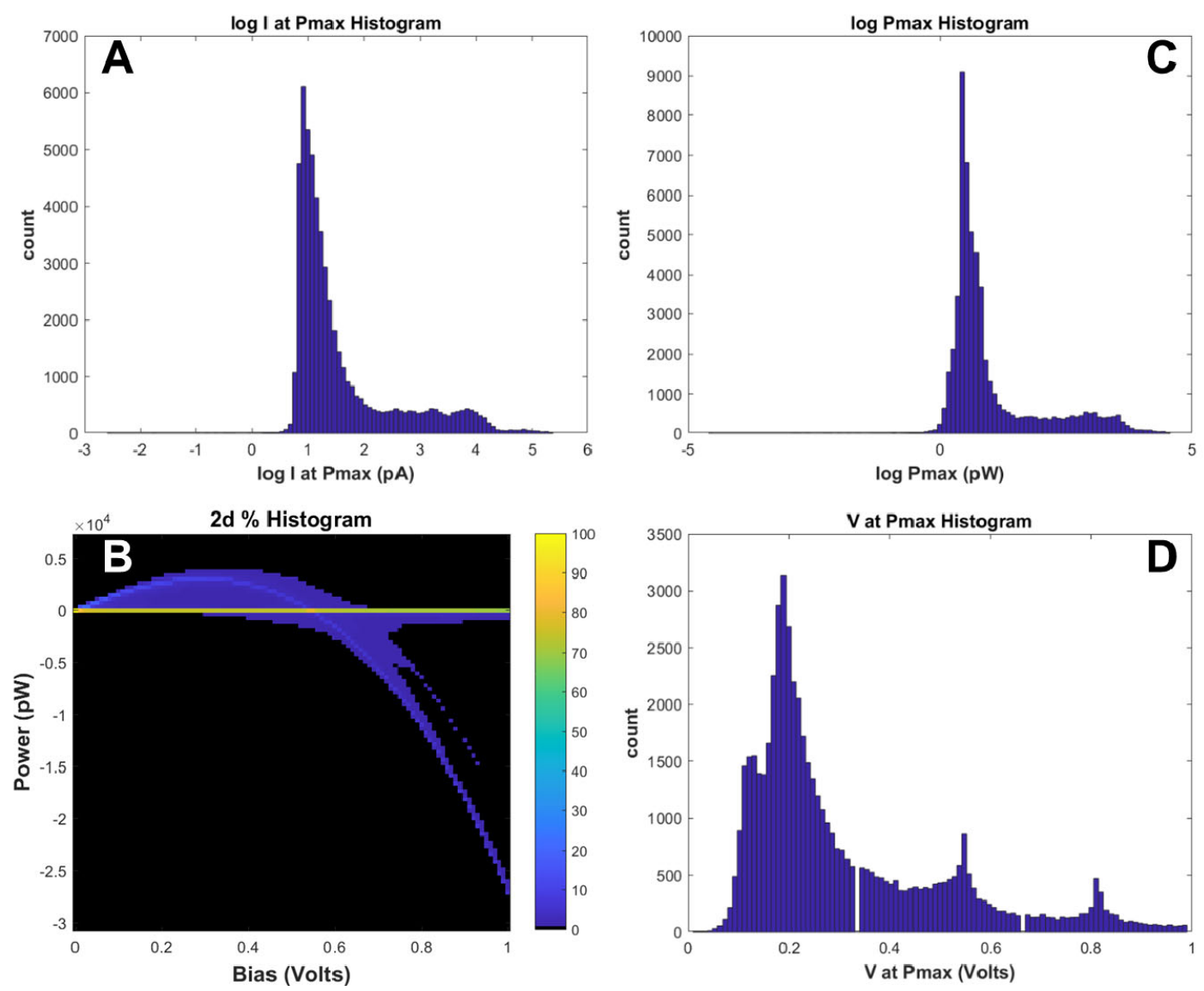

Fig. 6: Arrays of power versus voltage spectra, whether or not they are mapped, can provide statistics on (A) the current at the max power point, (B) the full swept behavior, (C) the max power itself, or (D) the bias at max power, demonstrating some of the potential insight to be afforded by eventual direct max power point mapping at the nanoscale.

Our direct $\mathrm{V}_{\mathrm{OC}}$ approach, and potentially direct max power mapping as well, are noteworthy as alternatives to prior AFM-based photovoltaic studies because of the possible combined advantages in acquisition time and spatial and voltage resolution. With respect to experiment 
time, for a standard line-scanning rate of $\sim 0.5 \mathrm{~Hz}$, one typical $256 \times 256$ pixel image directly mapping $V_{\text {oc }}$ will require $~ 8.5$ minutes to acquire. By comparison, our prior pc-AFMs work needs up to 1.4 hours for an equivalent resolution map. This assumes $2 \mathrm{~Hz}$ line rates (faster since there is 1 less feedback loop) during continuous imaging of 40 consecutive frames, each gradually revealing as many voltage steps for post-determination of locally calculated $V_{0 c}$ maps. The most common method for direct $V_{\text {Oc }}$ determination with AFM, on the other hand, is based on sequences of starts and stops in the AFM to collect individual I/V spectra. To maintain the same spatial resolution (65,536 pixels), this would necessitate a highly impractical 3.6-18.2 hours assuming a normal rate of 1-5 voltage sweeps per second, respectively. Finally, the other class of AFM-based measurements for determining $V_{o c}$, namely non-contact scanning surface potential mapping (also known as Kelvin probe), can often be acquired as quickly as our direct $V_{\text {oc }}$ approach, albeit with some unavoidable loss of spatial and voltage resolution since the measurement intrinsically detects the ensemble of possibly spatially varying surface potentials beneath the tip and cantilever when lifted above the surface [25]. In every one of these AFMbased cases, however, they are all susceptible to topographic artifacts as previously discussed, with particular sensitivity to variations in local curvature and obvious spatial drift concerns for slower measurement approaches.

Regardless of the approach utilized for $V_{o c}$, $I_{s c}$, or even maximum power mapping, understanding these parameters at the nanoscale can be instrumental in optimizing solar cell designs and processing of the rear passive emitter, vias, and back surface fields. Direct $V_{\text {oc }}$ or max power mapping, along with cross-correlations between these or other segmentable behaviors, may be especially valuable for photovoltaic degradation studies [26], either following real-world exposure, or based on standard accelerated aging protocols integrating thermal and humidity cycling, high intensity light soaking, chemical exposure, and/or various load conditions. For instance, equivalent measurements for sister specimens exposed to varying degrees of accelerated degradation [27], and then identical sectioning procedures especially for regions of interest identified by more macroscopic means (module I/V, imaged photoluminescence, etc.), could thereby enable site-specific investigations of such potential but unpredictable failure sites. This is especially important with PERC solar cells as the vias may be the most susceptible to chemical, compositional, or heat induced damage during the lifetime of PERC systems due to current crowding and the local lack of passivation [16, 28-30]. More broadly, degradation sites are also anticipated at current concentrating points for other photovoltaics, such as certain layer interfaces, grain boundaries, or planar defects in thin film or perovskite based systems [31-36]. 


\section{CONCLUSION}

A new method is demonstrated for nanoscale resolution mapping of the open circuit potential $\left(\mathrm{V}_{\mathrm{OC}}\right)$ in solar cells, specifically at the rear electrode interface for Passivated Emitter Rear Contact (PERC) cells but generally applicable to any photovoltaic. This is based on standard conducting AFM, but incorporates an extra feedback loop to continually update the solar cell electrode bias in order to always maintain zero current (i.e. $V_{O C}$ conditions). With the conducting AFM probe acting as a local back electrode during scanning, variability in the magnitude and spatial extent of $V_{O c}$ can thereby be uniquely assessed. This is analyzed alongside more common short circuit current (Isc) mapping in the vicinity of an individual via through the passive emitter rear contact, exposed by shallow-angle-cross-sectioning. The Al doped $p+/ p$ local back surface field is thereby directly detected and found to uniformly extend $\sim 2$ um into the underlying $\mathrm{p}$-type $\mathrm{Si}$ absorber. With such novel high resolution mapping of $\mathrm{V}_{\mathrm{Oc}}$, it becomes uniquely possible to assess the sensitivity to degradation of these critical regions of PERC or other solar cells where relative current crowding occurs, since locally higher temperatures and environment-induced molecular ingress are potential concerns for long term reliability.

\section{ACKNOWLEDGEMENT}

This material is based upon work supported by the U.S. Department of Energy's Office of Energy Efficiency and Renewable Energy (EERE) under Solar Energy Technologies Office (SETO) Agreement Number DE-EE-0008172. The DuPont Silicon Valley Technology Center is recognized for fabricating the investigated monocrystalline PERC cells, including Shannon Dugan and Joe Scardera for details on the cell processing and providing Fig. 4C.

\section{CONFLICT OF INTEREST}

The authors have no conflicts of interest related to the work published herein. 


\section{REFERENCES}

[1] S Daliento, A Chouder, P Guerriero, et al. (2017) International Journal of Photoenergy 2017: 13. Doi:10.1155/2017/1356851

[2] RA Sinton, A Cuevas (1996) Applied Physics Letters 69: 2510. Doi:10.1063/1.117723

[3] T Trupke, RA Bardos, M Abbott, J Cotter (2005) Applied Physics Letters 87. Doi:10.1063/1.2034109

[4] BD Huey, J Luria, DA Bonnell (2019) in Hawkes PW, Spence JCH (eds)Springer Handbook of MicroscopySpringer International Publishing, Cambridge, UK

[5] EM Tennyson, JL Garrett, JA Frantz, et al. (2015) Advanced Energy Materials 5: 1501142. Doi:10.1002/aenm.201501142

[6] T Glatzel, DF Marrón, T Schedel-Niedrig, S Sadewasser, MC Lux-Steiner (2002) Applied Physics Letters 81: 2017. Doi:10.1063/1.1506205

[7] Y Kutes, BA Aguirre, JL Bosse, JL Cruz-Campa, D Zubia, BD Huey (2016) Progress in Photovoltaics: Research and Applications 24: 315. Doi:10.1002/pip.2698

[8] T Dullweber, J Schmidt (2016) IEEE Journal of Photovoltaics 6: 1366. Doi:10.1109/JPHOTOV.2016.2571627

[9] U Zastrow, L Houben, D Meertens, A Grohe, T Brammer, E Schneiderlöchner (2006) Applied Surface Science 252: 7082. Doi:https://doi.org/10.1016/j.apsusc.2006.02.114

[10] M Padmanabhan, K Jhaveri, R Sharma, et al. (2016) physica status solidi (RRL) - Rapid Research Letters 10: 874. Doi:10.1002/pssr.201600173

[11] JL Braid, TD Wager, A Longacre, BD Huey, RH French (2018) 2018 IEEE 7th World Conference on Photovoltaic Energy Conversion (WCPEC)IEEE, Hawaii

[12] T Luka, S Großer, C Hagendorf, K Ramspeck, M Turek (2016) Solar Energy Materials and Solar Cells 158: 43. Doi:https://doi.org/10.1016/j.solmat.2016.05.061

[13] MCCd Oliveira, ASA Diniz Cardoso, MM Viana, VdFC Lins (2018) Renewable and Sustainable Energy Reviews 81: 2299. Doi:https://doi.org/10.1016/j.rser.2017.06.039

[14] J Zhu, M Koehl, S Hoffmann, et al. (2016) Progress in Photovoltaics: Research and Applications 24: 1346. Doi:10.1002/pip.2793

[15] C Peike, S Hoffmann, P Hülsmann, et al. (2013) Solar Energy Materials and Solar Cells 116: 49. Doi:https://doi.org/10.1016/j.solmat.2013.03.022

[16] A Kraft, L Labusch, T Ensslen, et al. (2015) IEEE Journal of Photovoltaics 5: 736. Doi:10.1109/JPHOTOV.2015.2395146

[17] K Matsuda, T Watanabe, K Sakaguchi, M Yoshikawa, T Doi, A Masuda (2012) Japanese Journal of Applied Physics 51: 10NF07. Doi:10.1143/jjap.51.10nf07

[18] G Illya, V Handara, L Yujing, R Shivakumar, AS Budiman (2016) Procedia Engineering 139: 7. Doi:https://doi.org/10.1016/i.proeng.2015.09.233

[19] K Nakayashiki, J Hofstetter, AE Morishige, et al. (2016) IEEE Journal of Photovoltaics 6: 860. Doi:10.1109/JPHOTOV.2016.2556981

[20] Y Xu (2018) SNEC 12, International Energy Storage and Mobile New Energy Exhibition \& ConferenceSNEC, Shanghai, China

[21] KD Shetty, MB Boreland, V Shanmugam, et al. (2013) Energy Procedia 33: 70. Doi:https://doi.org/10.1016/i.egypro.2013.05.041 
[22] K Atamanuk, J Luria, BD Huey (2018) Beilstein Journal of Nanotechnology 9: 1802. Doi:10.3762/bjnano.9.171

[23] Y Kutes, Y Zhou, JL Bosse, J Steffes, NP Padture, BD Huey (2016) Nano Letters 16: 3434. Doi:10.1021/acs.nanolett.5b04157

[24] K Dressler, M Rauer, M Kaloudis, S Dauwe, A Herguth, G Hahn (2015) IEEE Journal of Photovoltaics 5: 70. Doi:10.1109/JPHOTOV.2014.2359745

[25] BD Huey, DA Bonnell (2000) Solid State Ionics 131: 51. Doi:https://doi.org/10.1016/S0167-2738(00)00621-4

[26] EM Tennyson, TAS Doherty, SD Stranks (2019) Nature Reviews Materials 4: 573. Doi:10.1038/s41578-019-0125-0

[27] RH French, MP Murray, W Lin, et al. (2011) IEEE 2011 EnergyTechIEEE, Cleveland, OH

[28] N Ahn, K Kwak, MS Jang, et al. (2016) Nature Communications 7: 13422. Doi:10.1038/ncomms13422

[29] A Badiee, IA Ashcroft, RD Wildman (2016) International Journal of Adhesion and Adhesives 68: 212. Doi:https://doi.org/10.1016/j.ijadhadh.2016.03.008

[30] FJ Pern, AW Czanderna (1992) Solar Energy Materials and Solar Cells 25: 3. Doi:https://doi.org/10.1016/0927-0248(92)90013-F

[31] J Luria, Y Kutes, A Moore, L Zhang, EA Stach, BD Huey (2016) Nature Energy 1: 16150. Doi:10.1038/nenergy.2016.150

[32] MS Leite, M Abashin, HJ Lezec, A Gianfrancesco, AA Talin, NB Zhitenev (2014) ACS Nano 8: 11883. Doi:10.1021/nn5052585

[33] S Sadewasser, D Abou-Ras, D Azulay, et al. (2011) Thin Solid Films 519: 7341. Doi:https://doi.org/10.1016/i.tsf.2010.12.227

[34] I Visoly-Fisher, SR Cohen, K Gartsman, A Ruzin, D Cahen (2006) Advanced Functional Materials 16: 649. Doi:10.1002/adfm.200500396

[35] JB Li, V Chawla, BM Clemens (2012) Advanced Materials 24: 720. Doi:10.1002/adma.201103470

[36] SA Galloway, PR Edwards, K Durose (1999) Solar Energy Materials and Solar Cells 57: 61. Doi:https://doi.org/10.1016/S0927-0248(98)00168-8 